\author{
Marquette University \\ e-Publications@Marquette
}

Biomedical Engineering Faculty Research and

Publications

Biomedical Engineering, Department of

2012

\title{
Exploring Functional Networks of the Brain Relating to Upper Extremity Motor Skill Using Graph Theory
}

Dominic Nathan

Marquette University

Stephen Guastello

Marquette University, stephen.guastello@marquette.edu

Robert W. Prost

Medical College of Wisconsin

Dean C. Jeutter

Marquette University

Follow this and additional works at: https://epublications.marquette.edu/bioengin_fac

Part of the Biomedical Engineering and Bioengineering Commons

\section{Recommended Citation}

Nathan, Dominic; Guastello, Stephen; Prost, Robert W.; and Jeutter, Dean C., "Exploring Functional Networks of the Brain Relating to Upper Extremity Motor Skill Using Graph Theory" (2012). Biomedical Engineering Faculty Research and Publications. 62.

https://epublications.marquette.edu/bioengin_fac/62 
initiate phase transition from conscious individuality and logical thought to emergency subconscious routines and collective reactions. It is hypothesised perception of signals consistent with the presence of danger initiate rapid activation of fight or flight responses favouring survival in riot situations. Activation of this physiological collective communication is hypothesised to only be initiated in emotionally charged situations due to a greater metabolic cost detection incurs. Evolutionary advantage can be inferred in those with sensitivity to perceiving them. Emotional emissions are argued to be amplified or damped to a greater or lesser degree by the interaction of ambient magnetic fields from the earth, individual physiological differences and the variable impact of solar wind fluctuations on the magnetosphere and atmospheric Schumann resonation field.

Barbara Meeker, Sociology, University of Maryland.

\section{Nonlinear Dynamics of Group Dynamics: Models from Mathematical Sociology}

I present a summary of a research program in which assumptions from the classic Lotka-Volterra model of species competition are adapted to apply to the development of inequalities in amount of contribution to a group discussion. This kind of mathematical system is well known to be very sensitive to the initial values of the parameters, with parameters in one range producing output of two actors that converges while when parameters are in another range one actor"s output falls to zero and the other reaches a stable positive result. To adapt this model to human interaction, I take into account that human beings monitor and adjust their behavioral output throughout an interaction. This requires an additional assumption, that the parameters vary depending on how unequal the rates of contribution have become. Since this introduces a feedback loop that prevents direct solution of equations, I use a computer simulation to develop predictions. Different values of initial parameters produce some results that stabilize at equality, others that stabilize at unequal rates of output, and still others that seem chaotic. Of most interest to sociologists are the ones that stabilize at unequal (but not zero) rates of participation. These results are expanded to apply to groups of three, four, or more members and compared with data from actual discussion groups.

Dominic Nathan, Department of Biomedical

Engineering, Marquette University

Stephen Guastello, Department of Psychology, Marquette University

Robert Prost, Department of Radiology, Medical College of Wisconsin

Dean J eutter, Department of Biomedical Engineering, Marquette University

\section{Exploring Functional Networks of the Brain Relating to Upper Extremity Motor Skill Using Graph Theory}

Connectivity analysis of the brain provides a method of identifying networks that relate to functional and anatomical pathways. The ability to quantify functional brain networks has been critical for the detection of various neurocognitive disorders such as ADHD, Alzheimer s disease and schizophrenia. However the majority of research has been conducted on resting state data sets which are absent of explicit stimuli. The examination of network connectivity relating to specific tasks such as hand use could add benefit to understanding recovery, cortical reorganization and transfer of skill, especially in individuals suffering from chronic physical dysfunction such as stroke, spinal cord or traumatic brain injury. The efficiency of information flow, network structure, integrity and the interaction among anatomical regions of the brain that are specific to the performance of voluntary upper extremity motor tasks were investigated. In this paper, we examine functional connectivity of the brain using complex network analysis tools of graph theory. More specifically, we examine functional networks and their characteristics relating to motor skill during the performance of tasks using the dominant and non-dominant hand. Analysis was performed using data from a custom developed time resolved fMRI paradigm involving human subjects $(\mathrm{N}=5)$. A total of 5 measurements were calculate including the cluster coefficient, efficiency, density, characteristic path length and degrees for inter region and within region networks. The networks analyzed were weighted and undirected. The results provide quantitative information regarding the interactions of brain regions that are involved with the functional motor tasks. Furthermore the results provide insight regarding local, within-region organization of networks which have implications of neural correlates relating to motor skill. Information from this study has potential for the development of quantitative neuro markers which may provide further insight regarding plasticity and cortical reorganization from injury and treatment.

David Pincus, Kiersten Eberle, Christin S. Walder, Crean School of Health and Life Sciences, Chapman University

Curt A. Sandman, Aaron S. Kemp, Christopher Mabini, Department of Psychiatry and Human Behavior University of California, Irvine School of Medicine

\section{Intentional Self-harm and Behavioral Resilience}

There is no commonly accepted explanation or intervention for severe and persistent self-injurious behavior (SIB). This study utilized orbital decomposition to examine the impacts of SIB on the dynamics of behavior-environmental interactions over time. Methods: Data consisted of categorical time-series of sequential behaviors gathered from individuals within a residential treatment setting for adults with developmental disabilities along with severe and persistent SI B. Several 2.5 hour series were collected for each participant, some with SIB $(\mathrm{N}=134)$ and some without (Non-SIB; $N=$ 96). Results: (1) Series were characteristic of selforganizing systems (mean Lyapunov Dimension $=1.2$; range: 13.64 with $98 \%$ of values lower than 2.0 ). (2) 